Audiology

Neurotology
Audiol Neurotol 2012;17:189-197

DOI: $\underline{10.1159 / 000336407}$
Received: May 9, 2011

Accepted after revision: January 9, 2012

Published online: March 3, 2012

\title{
Psychoacoustic Performance and Music and Speech Perception in Prelingually Deafened Children with Cochlear Implants
}

\author{
Kyu Hwan Jung a, e Jong Ho Won ${ }^{a, b}$ Ward R. Drennan ${ }^{a}$ Elyse Jameyson ${ }^{a}$ \\ Gary Miyasaki $^{a, b}$ Susan J. Norton ${ }^{a, d}$ Jay T. Rubinstein ${ }^{a, c, d}$ \\ aVirginia Merrill Bloedel Hearing Research Center, Department of Otolaryngology - Head and Neck Surgery \\ and Departments of ${ }^{b}$ Speech and Hearing Sciences and ${ }^{\mathrm{C} B i o e n g i n e e r i n g, ~ U n i v e r s i t y ~ o f ~ W a s h i n g t o n, ~ a n d ~}$ \\ ${ }^{\mathrm{d}}$ Divisions of Audiology and Otolaryngology, Seattle Children's Hospital, Seattle, Wash., USA; ${ }^{\text {eDepartment of }}$ \\ Otorhinolaryngology - Head and Neck Surgery, Inje University, Haeundae Paik Hospital, Busan, Korea
}

\section{Key Words}

Cochlear implant - Prelingual hearing loss $\cdot$ Psychophysics • Music $\cdot$ Speech perception

\begin{abstract}
The number of pediatric cochlear implant $(\mathrm{Cl})$ recipients has increased substantially over the past 10 years, and it has become more important to understand the underlying mechanisms of the variable outcomes in this population. In this study, psychoacoustic measures of spectral-ripple and Schroeder-phase discrimination, the Clinical Assessment of Music Perception, and consonant-nucleus-consonant (CNC) word recognition in quiet and spondee reception threshold (SRT) in noise tests have been presented to 11 prelingually deafened $\mathrm{Cl}$ users, aged 8-16 years with at least 5 years of $\mathrm{Cl}$ experience. The children's performance was compared to the previously reported results of postlingually deafened adult $\mathrm{Cl}$ users. The average spectral-ripple threshold $(n=10)$ was 2.08 ripples/octave. The average Schroeder-phase discrimination was $67.3 \%$ for $50 \mathrm{~Hz}$ and $56.5 \%$ for $200 \mathrm{~Hz}(\mathrm{n}=9)$. The Clinical Assessment of Music Perception test showed that the average complex pitch direction discrimination was 2.98 semitones. The mean melody score was at a chance level, and the mean timbre score was $34.1 \%$ correct. The mean CNC word
\end{abstract}

recognition score was $68.6 \%$, and the mean SRT in steady noise was $-8.5 \mathrm{~dB}$ SNR. The children's spectral-ripple resolution, CNC word recognition, and SRT in noise performances were, within statistical bounds, the same as in a population of postlingually deafened adult $\mathrm{Cl}$ users. However, Schroeder-phase discrimination and music perception were generally poorer than in the adults. It is possible then that this poorer performance seen in the children might be partly accounted for by the delayed maturation in their temporal processing ability, and because of this, the children's performance may have been driven more by their spectral sensitivity.

Copyright $\odot 2012$ S. Karger AG, Basel

\section{Introduction}

A number of studies have investigated speech and music perception and a variety of psychoacoustic performance metrics in postlingually deafened adult cochlear implant (CI) listeners. However, relatively few studies have been reported investigating outcomes in prelingually deafened children using CIs [Chen et al., 2010]. The number of pediatric cochlear implant recipients has been markedly increasing over the past 10 years [Bradham and Jones, 2008], but speech perception outcomes still vary

\section{KARGER}

Fax +4161306 1234

E-Mail karger@karger.ch

www.karger.com (c) 2012 S. Karger AG, Basel

$1420-3030 / 12 / 0173-0189 \$ 38.00 / 0$

Accessible online at:

www.karger.com/aud
Jong Ho Won, PhD

Virginia Merrill Bloedel Hearing Research Center

Department of Otolaryngology - Head and Neck Surgery, University of Washington Seattle, WA 98195 (USA)

Tel. +1 206616 2041, E-Mail jhwon@uw.edu 
greatly. This is partly due to the fact that speech perception involves multiple interactions between temporal and spectral acoustic information of the speech signals. Therefore, it is important to understand what acoustic cues drive clinical success in implanted children. At the same time, it is crucial to understand how implanted children differ from postlingually deafened implanted adults so that better sound-encoding strategies, mapping, and rehabilitation paradigms can be developed for this population.

Previous studies have evaluated the contribution of spectral and temporal sensitivity to speech or music perception performance in postlingually deafened adult CI listeners [Fu, 2002; Henry and Turner, 2003; Henry et al., 2005; Won et al., 2007, 2010, 2011; Drennan et al., 2008; Saoji et al., 2009]. For example, spectral-ripple discrimination has been widely used to evaluate the spectral sensitivity in CI users and its relationship with speech perception. The spectral-ripple test evaluates listeners' ability to discriminate between standard and inverted ripple spectrum. The stimuli are composed of frequency components in which their spectral peaks and valleys are interchanged over a wide range of frequency. Spectralripple thresholds determined by the minimum ripple spacing discriminated by listeners have been shown to correlate with vowel and consonant recognition [Henry and Turner, 2003; Henry et al., 2005], word recognition in quiet and in noise [Won et al., 2007], and music perception in postlingually deafened adult CI users [Won et al., 2010]. To our best knowledge, spectral-ripple discrimination in prelingually deafened children wearing CIs has not been reported.

A recent study by Drennan et al. [2008] used Schroeder-phase discrimination to evaluate CI users. Positive and negative Schroeder-phase stimuli have nearly flat temporal envelopes and identical long-term spectra but a different temporal fine structure [Schroeder, 1970]. Thus, Schroeder-phase discrimination has been used to evaluate the acoustic temporal fine structure sensitivity in normal-hearing and hearing-impaired listeners [Summers and Leek, 1998; Summers, 2000; Oxenham and Dau, 2001; Dooling et al., 2002; Lauer et al., 2009]. Drennan et al. [2008] demonstrated that Schroeder-phase discrimination evaluates the ability of CI users to track temporal modulations that sweep rapidly across channels. This discrimination performance had a small but significant correlation with speech perception in CI users.

The present study evaluated the spectral and temporal sensitivity of prelingually deafened implanted children using spectral-ripple discrimination and Schroederphase discrimination. The assessment of these two met- rics allows the evaluation of spectral and temporal sensitivity in such children and further advances our understanding of the similarities and differences between prelingually deafened children and postlingually deafened adults in their speech and music perception abilities. The primary goal of the present study was to measure spectral and temporal sensitivity, speech perception abilities in quiet and in background noise, and music perception abilities in prelingually deafened children with CIs and compare the results to published data on adults who were postlingually deafened and subsequently implanted. To measure speech perception abilities, open-set monosyllabic word recognition in quiet and closed-set spondee word recognition in steady-state noise were evaluated. To determine music perception abilities, the Clinical Assessment of Music Perception (CAMP) test was performed. Previous work has shown that all of these tests are reliable, showing good test-retest reliability with minimal learning in adult CI users [Won et al., 2007; Drennan et al., 2008; Kang et al., 2009]. The secondary goal was to examine relationships between the spectral/ temporal sensitivities and speech and music perception abilities.

\section{Methods}

Subjects

Eleven prelingually deafened CI users were recruited from Seattle Children's Hospital (7 males and 4 females). Their ages ranged from 8 to 16 years $($ mean $=12.1)$. The subject recruitment criteria included implantation before the age of 5 years (mean $=$ 2.4 ), more than 5 years of CI use (range $=5-16$ ), and age of $8-16$ years at testing. All subjects were native English speakers and bilaterally deafened and had no residual hearing in either ear. They were all normally developing children except for their hearing loss. Detailed subject information is listed in table 1 . The use of human subjects in this study was reviewed and approved by the Seattle Children's Hospital Institutional Review Board. Some subjects did not participate in all experiments due to time and scheduling constraints.

\section{Test Administration}

All subjects listened to the stimuli using their own sound processor set to a comfortable listening level. Bilateral users were tested with their first implanted ear only. None of the subject used a hearing aid. CI sound processor settings were not changed from the clinical settings throughout all tests. All tests were conducted in a double-walled, sound-treated booth (IAC). Custom MATLAB (The Mathworks, Inc.) programs were used to present stimuli with a Crown D45 amplifier. A single loudspeaker (B\&W DM303), positioned $1 \mathrm{~m}$ from the subjects at zero degrees azimuth, presented stimuli in sound field. The order of test administration was randomized across the subjects. The duration of the entire test battery for one subject was approximately $2 \mathrm{~h}$. Most 
Table 1. Demographics of prelingually deafened test subjects

\begin{tabular}{|c|c|c|c|c|c|c|c|c|}
\hline $\begin{array}{l}\text { Subject } \\
\text { ID }\end{array}$ & $\begin{array}{l}\text { Age at } \\
\text { test, years }\end{array}$ & Gender & $\begin{array}{l}\text { Age at implant } \\
\text { years } \\
\text { (contralateral) }\end{array}$ & $\begin{array}{l}\text { Duration of } \\
\text { CI use, years } \\
\text { (contralateral) }\end{array}$ & Etiology & Device & Processor & $\begin{array}{l}\text { Current educational } \\
\text { environment }\end{array}$ \\
\hline K001J & 10 & M & 4 & 6 & Connexin 26 & CII & F120 & $\mathrm{m}$ \\
\hline K002J & 12 & M & 3 & 9 & Connexin 26 & $\mathrm{~N} 24$ & $\mathrm{ACE}$ & $m$ with sign interpretation \\
\hline K003J & 9 & M & 1 & 9 & unknown & CII & F120 & $\mathrm{m}$ \\
\hline K004T & 8 & M & $1(6)$ & $7(2)$ & unknown & N24/freedom & $\mathrm{ACE}$ & $\mathrm{m}$ \\
\hline K005J & 15 & $\mathrm{~F}$ & 3 & 12 & CMV & $\mathrm{N} 22$ & SPEAK & $\mathrm{m}$ with captioning \\
\hline K007J & 11 & M & 2 & 9 & unknown & $\mathrm{N} 24$ & $\mathrm{ACE}$ & $\mathrm{m}$ \\
\hline $\mathrm{K} 008 \mathrm{~N}$ & 12 & $\mathrm{~F}$ & 3 & 9 & unknown & $\mathrm{N} 24$ & $\mathrm{ACE}$ & $\mathrm{m}$ \\
\hline K009A & 13 & $\mathrm{~F}$ & $2(10)$ & $11(2)$ & Connexin 26 & N24/freedom & $\mathrm{ACE}$ & $\mathrm{nm}$ \\
\hline K010E & 14 & $\mathrm{~F}$ & $3(12)$ & $11(2)$ & Connexin 26 & N24/freedom & $\mathrm{ACE}$ & $\mathrm{m}$ with interpretation \\
\hline $\mathrm{K} 011 \mathrm{H}$ & 16 & M & 2 & 14 & hypoxia (placenta abruption) & $\mathrm{N} 22$ & SPEAK & $\mathrm{m}$ \\
\hline S013A* & 13 & $\mathrm{~F}$ & $2(9)$ & $11(3)$ & meningitis & $90 \mathrm{k}$ & F120 & $\mathrm{m}$ \\
\hline
\end{tabular}

$\mathrm{m}=$ Mainstream education; $\mathrm{nm}=$ school for hearing impaired; CMV = cytomegalovirus infection.

* This patient was originally implanted 11 years ago with a CII device that failed. She has had the right ear explanted and re-implanted two more times. She was using her third internal device on the right side at the test time.

children (all except for K001J, K002J, K003J, and K004T) finished the entire test in a day within $2 \mathrm{~h}$.

\section{Spectral-Ripple Discrimination Test}

The spectral-ripple discrimination test used the same stimuli and threshold estimation procedure described by Won et al. [2007]. The ripple stimuli were generated by summing 200 puretone frequency components. The stimuli had a total duration of $500 \mathrm{~ms}$ and were ramped with a rise/fall time of $150 \mathrm{~ms}$. Each 500 ms stimulus was either a standard (reference stimulus) or inverted ripple (ripple phase-reversed test stimulus). For standard ripples, the phase of the full-wave rectified sinusoidal spectral envelope was set to 0 radians, and for inverted ripples to $\pi / 2$. The amplitudes of the components were determined by a full-wave rectified sinusoidal envelope on a logarithmic amplitude scale. The ripple peaks were spaced equally on a logarithmic amplitude scale. The stimuli had a bandwidth of $100-5000 \mathrm{~Hz}$ and a peak-to-valley ratio of $30 \mathrm{~dB}$. The mean presentation level of the stimuli was $61 \mathrm{dBA}$ and randomly roved by $\pm 4 \mathrm{~dB}$ in 1 - $\mathrm{dB}$ steps. The starting phases of the components were randomized for each presentation. The ripple densities differed by ratios of 1.414 and included $0.125,0.176$, $0.250,0.354,0.500,0.707,1.000,1.414,2.000,2.828,4.000,5.657$, 8.000 , and 11.314 ripples/octave. A 3-interval, adaptive forcedchoice (AFC) paradigm using a two-up and one-down adaptive procedure was used to determine spectral-ripple discrimination thresholds, converging on $70.7 \%$ correct [Levitt, 1971]. Feedback was not provided. The threshold for a single adaptive track was determined by averaging the ripple spacing (the number of ripples/ octave) for the final 8 of 13 reversals. The final threshold value for each subject was determined as the mean of the thresholds across three test runs. Prior to actual testing, to ensure the subjects were familiar with the stimuli and the task, they listened to few example stimuli in the presence of the experimenter.

Schroeder-Phase Discrimination Test

The Schroeder-phase discrimination test used in the current study is the same as that previously described by Drennan et al.
[2008]. Positive and negative Schroeder-phase stimulus pairs were created for two different fundamental frequencies (F0s) of 50 and $200 \mathrm{~Hz}$. These frequencies were chosen because the 50- and 200$\mathrm{Hz}$ scores can assess a wide range of CI listeners' performances on the test and because they correlate with a variety of clinical outcome measures [Drennan et al., 2008]. For each F0, equal-amplitude cosine harmonics from the $\mathrm{F} 0$ up to $5 \mathrm{kHz}$ were summed. Phase values for each harmonic were determined by the equation $\theta_{n}= \pm \pi n(n+1) / N$, where $\theta_{n}$ is the phase of the nth harmonic, $n$ is the nth harmonic, and $N$ is the total number of harmonics in the complex. The Schroeder-phase stimuli were presented at 65 $\mathrm{dBA}$ without roving the level. A 4-interval, 2-AFC procedure was used. One stimulus (i.e. positive Schroeder-phase, test stimulus) occurred in either the second or third interval and was different from three others (i.e. negative Schroeder phase, reference stimulus). The subject's task was to discriminate the test stimulus from the reference stimuli. To determine a total percent correct for each F0, the method of constant stimuli was used. In a single test block, each $\mathrm{F} 0$ was presented 24 times in random order, and a total percent correct for each F0 was calculated as the percent of stimuli correctly identified. The final score for this test was the mean percent correct of four test blocks for each F0. The total number of presentations for each F0 was 96. Visual feedback was provided. Prior to actual testing, the subjects listened to the example stimuli two times for each frequency.

The Consonant-Nucleus-Consonant Word Recognition Test

Fifty consonant-nucleus-consonant (CNC) monosyllabic words (e.g. Home, June, Pad, Sun) from a recorded CD with a male talker were presented from an open set in sound field at $65 \mathrm{dBA}$ [Peterson and Lehiste, 1962]. Each CNC word list was randomly chosen out of ten lists for each subject. A total percent correct score was calculated as the percent of words correctly repeated. Feedback was not provided. 
Table 2. CNC word recognition test score

\begin{tabular}{ll}
\hline Subject ID & CNC word (\% correct) \\
\hline K001J & 64 \\
K002J & 46 \\
K003J & 80 \\
K004T & N/A \\
K005J & 52 \\
K007J & 72 \\
K008N & 88 \\
K009A & 82 \\
K010E & 50 \\
K011H & 80 \\
S013A & 72 \\
Mean & $68.6( \pm 14.9)$ \\
Mean for adults* & $68.9( \pm 18.1)$ \\
\hline
\end{tabular}

* Adult data $(\mathrm{n}=15)$ adopted from Won et al. [2007].

Speech Reception Threshold in Steady Noise Test

The procedure for administering the speech reception threshold (SRT) test was the same as that previously described by Won et al. [2007]. The subjects were asked to identify one randomly chosen spondee word out of a closed set of 12 equally difficult spondees [Harris, 1991] in the presence of background noise [Turner et al., 2004]. The spondees, two-syllable words with equal emphasis on each syllable, were recorded by a female talker. A steady-state, speech-shaped background noise was used, and the onset of the spondees was $500 \mathrm{~ms}$ after the onset of the background noise. The steady-state noise had a duration of $2.0 \mathrm{~s}$. The level of the target speech was $65 \mathrm{dBA}$. The level of the noise was tracked using a one-down one-up procedure with 2-dB steps. Feedback was not provided. For all subjects, the adaptive track started with a $+10 \mathrm{~dB}$ signal-to-noise ratio (SNR) condition. The threshold for a single test run was estimated by averaging the SNR for the final 10 of 14 reversals. The subjects completed three test runs, and the final threshold for each subject was determined as the mean of the thresholds across these three test runs.

The CAMP Test

The CAMP was used for music testing. The procedure for administering the CAMP test was the same as that previously described by Nimmons et al. [2008] and Kang et al. [2009]. Three components of music perception (pitch, melody, and timbre recognition) were assembled into a computer-driven exercise. Each subtest began with a brief training session in which the subjects were able to familiarize themselves with the test items and protocol.

Complex-Pitch Direction Discrimination. The pitch direction discrimination task was a 2-AFC procedure using synthesized complex tones, and required subjects to identify which of the two complex tones has the higher F0. The dependent variable was the justnoticeable-difference threshold in semitones determined using a one-up one-down tracking procedure. The threshold was estimated as the mean interval size for three repetitions, each determined from the mean of the final 6 of 8 reversals. Complex tones of three F0s $(262,330$, and $392 \mathrm{~Hz})$ were used in the task, and the primary dependent variable was the average of the threshold in semitones for all fundamental frequencies. The validity of this approach is further discussed in Kang et al. [2009] or Won et al. [2010].

Melody Identification. In the melody recognition subtest, 12 well-known melody clips were played three times in random order. Rhythm cues were removed such that notes of a longer duration were repeated in a $1 / 8$-note pattern. The presentation level was $65 \mathrm{dBA}$, and the amplitude of each note was randomly roved by $4 \mathrm{~dB}$. The melody test used synthesized piano-like tones which have identical envelopes. Subjects were asked to identify melodies by selecting the title corresponding to the melodies presented. The primary dependent variable was a total percent correct score calculated after 36 melody presentations including 3 presentations of each melody.

Timbre Identification. In the timbre (musical instrument) recognition subtest, 8 musical instruments, each playing the same recorded melody composed of five notes, were presented three times in random order. The instruments were the piano, trumpet, clarinet, saxophone, flute, violin, cello, and guitar. The presentation level was $65 \mathrm{dBA}$. The subjects were instructed to select the labeled icon of the instrument corresponding to the timbre presented. The primary dependent variable was a total percent correct score calculated after 24 presentations including 3 presentations of each instrument.

Data Analysis

Data for postlingually deafened adults CI listeners were adopted from previous publications: Won et al. [2007] for spectralripple discrimination thresholds, CNC word recognition scores, SRTs in steady-state noise; Drennan et al. [2008] for Schroederphase discrimination scores, and Kang et al. [2009] for CAMP test scores. Student's t test was used to compare the mean scores between the child and adult CI listeners. For the child data in the present study, correlation analysis was performed to determine if spectral-ripple and Schroeder-phase discriminations correlate with speech and music outcomes. Pearson's correlation coefficients are reported. Error bars in all figures indicate 95\% confidence intervals across the subjects.

\section{Results}

Ages (mean $=12.1$ years) and duration of CI usage (mean $=9.8$ years) were not correlated with any of the test results. There were no differences in gender and educational level.

\section{Spectral-Ripple Discrimination}

Spectral-ripple thresholds for individual subjects are shown in figure 1 . The mean spectral-ripple threshold for 10 child subjects was $2.08 \pm 1.6$ ripples/octave $( \pm 95 \%$ confidence interval). A t test indicated that there was no statistical difference $(\mathrm{p}=0.4)$ between the results of the children in the present study and the adult average of 1.73 \pm 0.9 ripples/octave reported by Won et al. [2007]. 
Fig. 1. Spectral-ripple discrimination thresholds. Error bars indicate 1 SD. K004T did not finish the test. ${ }^{*}$ Adult data $(\mathrm{n}=31)$ adopted from Won et al. [2007].
Fig. 2. Schroeder-phase discrimination test scores. The mean scores for the 50 and $200-\mathrm{Hz}$ Schroeder-phase stimuli in children were worse than in adults $(\mathrm{p}=$ 0.012 for $50 \mathrm{~Hz}$; $\mathrm{p}=0.046$ for $200 \mathrm{~Hz}$ ). K001J and K003J did not conduct the test. Error bars indicate 1 SD. ${ }^{*}$ Adult data $(\mathrm{n}=$ 24) adopted from Drennan et al. [2008].
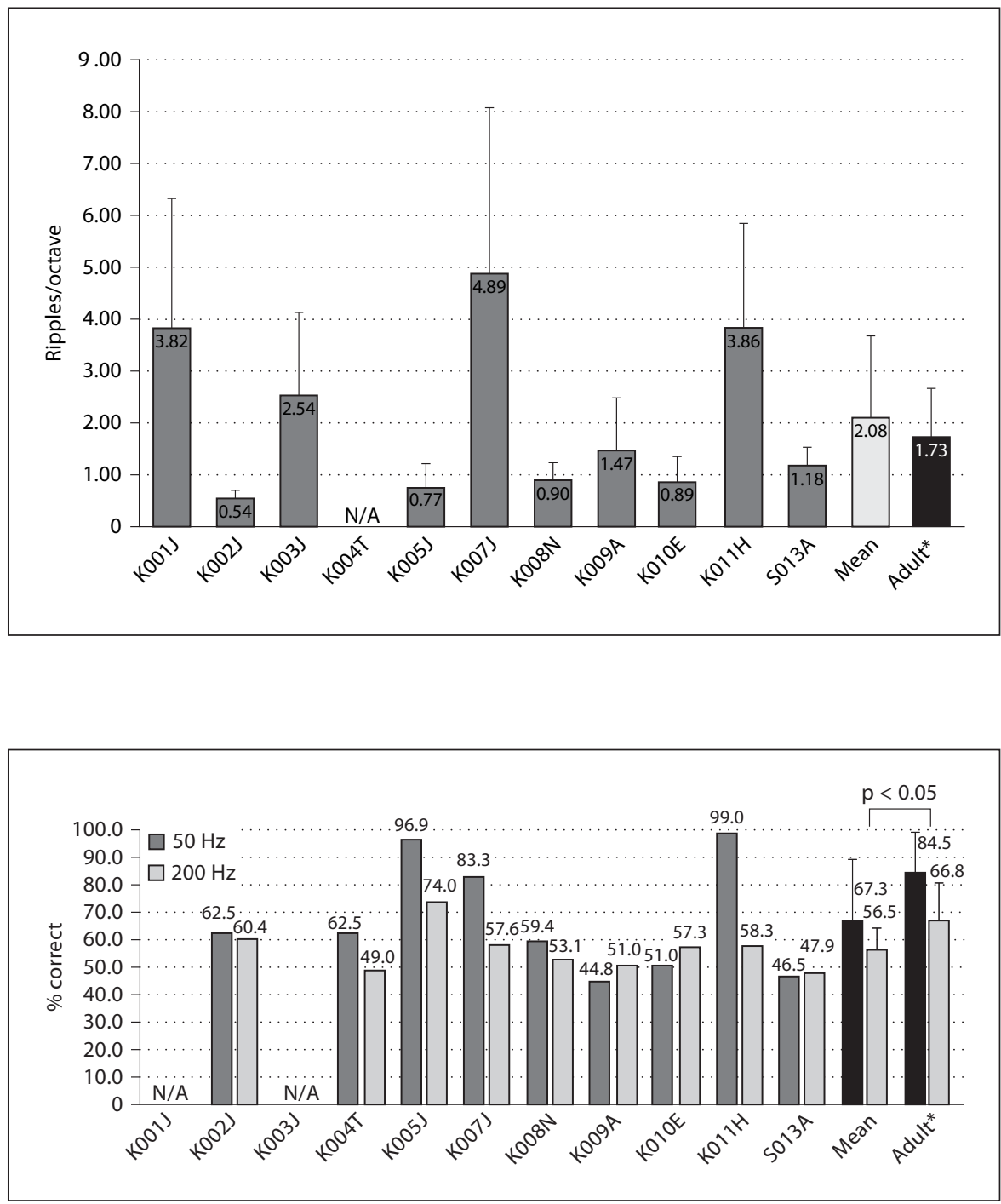

\section{Schroeder-Phase Discrimination}

For 9 children, the average score for the $50-\mathrm{Hz}$ Schroeder-phase discrimination was $67.3 \pm 20.8 \%$ and for the $200-\mathrm{Hz}$ Schroeder-phase discrimination $56.5 \pm$ $7.9 \%$. Individual scores are shown in figure 2. For 24 adult CI subjects, Drennan et al. [2008] reported mean scores of $84.5 \%$ for $50 \mathrm{~Hz}$ and $66.8 \%$ for $200 \mathrm{~Hz}$. The 50 - and $200-\mathrm{Hz}$ Schroeder-phase scores for children were above the chance level $(\mathrm{p}=0.04)$, and when compared to the adult subjects, child subjects were worse at both frequencies $(\mathrm{p}=0.01$ for $50 \mathrm{~Hz}$; $\mathrm{p}=0.046$ for $200 \mathrm{~Hz})$. This result was also confirmed by a repeated measure of ANOVA. The effect of Schroeder-phase frequency (as a withinsubject variable, $\mathrm{F}[1,30]=26.1, \mathrm{p}<0.0001)$ and subject populations (i.e. children vs. adults as a between-subject variable, $F[1,30]=6.6, p=0.016)$ was significant, whereas there was no interaction between the two factors $(\mathrm{F}[1,30]=101.2, \mathrm{p}=0.32)$.

\section{CNC Word Recognition}

Table 2 shows CNC word recognition scores for individual subjects. The average $\mathrm{CNC}$ word recognition score for 10 child subjects was $68.6 \pm 14.9 \%$, which is nearly identical to the average CNC score for 15 adult subjects $(68.9 \pm 18.1 \%)$ reported by Won et al. [2007] and similar to the score of $63 \pm 4.3 \%$ for an unpublished cohort of 97 postlingually deafened implanted adults. 
Fig. 3. SRT scores in the steady state noise test. Mean SRT scores of children were not different from those of adults $(\mathrm{p}=0.67)$. Error bars indicate 1 SD. * Adult data $(\mathrm{n}=$ 29) adopted from Won et al. [2007].

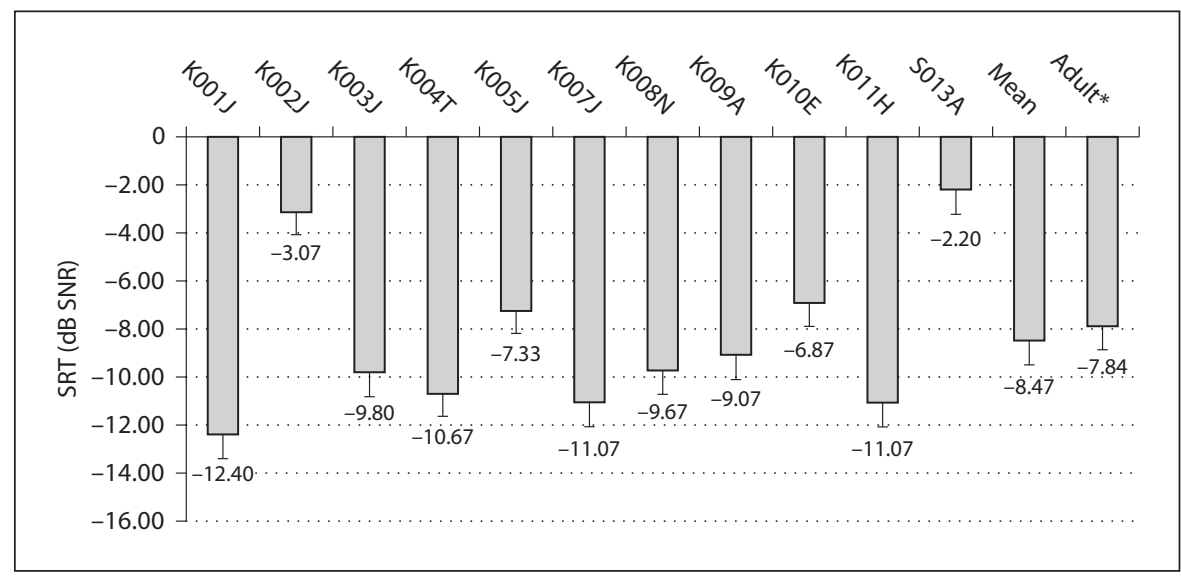

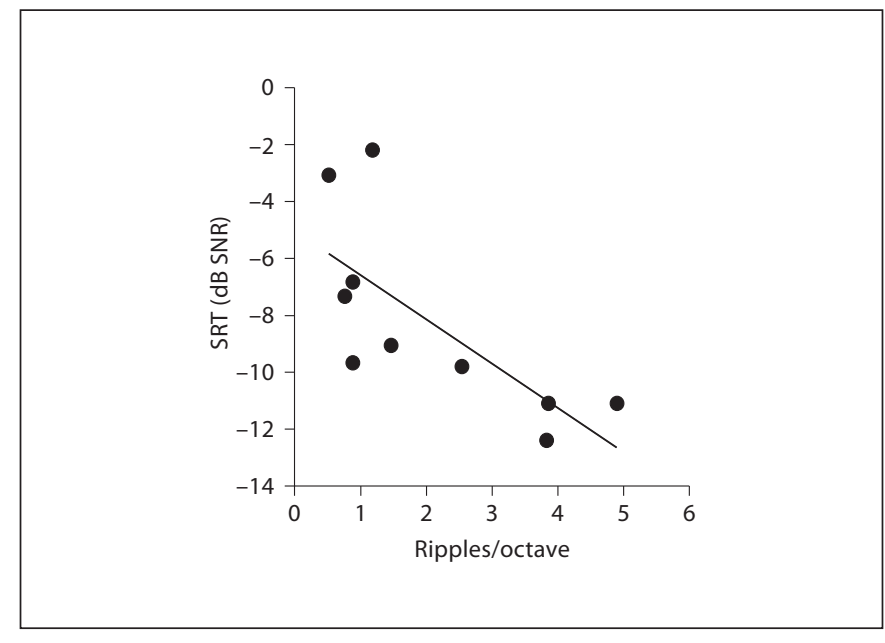

Fig. 4. Scatter plot between spectral-ripple discrimination thresholds and SRTs in steady noise. The solid line represents linear regression $(\mathrm{r}=-0.72, \mathrm{p}=0.018)$. Note that the $\mathrm{p}$ value is not corrected for multiple analyses.

\section{SRT in Steady Noise}

The average SRT for all 11 children was $-8.5 \pm 3.3 \mathrm{~dB}$ SNR. Individual SRTs for these subjects are shown in figure 3. Previously, the mean SRT for 29 adult CI subjects was reported to be $-7.8 \pm 4.47 \mathrm{~dB}$ SNR. As with CNC word recognition, SRTs in the two populations were very similar. In the 11 children, a significant correlation was found between CNC scores and SRTs in noise $(r=-0.71$, $\mathrm{p}<0.001$ ). Won et al. [2007] showed a significant correlation between spectral-ripple thresholds and SRTs in steady-state noise for 29 adult CI subjects $(\mathrm{r}=-0.62, \mathrm{p}=$ $0.0004)$. For 10 child subjects in the present study, a significant correlation was also found between spectral-rip- ple thresholds and SRTs in steady-state noise $(r=-0.72$, $\mathrm{p}=0.018$ ). Figure 4 shows the scattergram of spectralripple thresholds and SRTs in steady-state noise.

\section{Clinical Assessment of Music Perception}

The total duration for all CAMP tests including pitch, melody, and timbre subtests was $25.3 \pm 4.6 \mathrm{~min}$. Pediatric implantees reported that the melody test was most difficult. However, all 11 subjects completed the subtests with minimal intervention from the test administrator.

\section{Complex-Pitch Direction Discrimination}

The complex-pitch direction discrimination ability of the children was the same as of the adult implantees $(\mathrm{p}=$ 0.96; fig. 5). The mean pitch direction threshold over three base frequencies was $2.98 \pm 2.23$ semitones. The pitch direction thresholds for the three base frequencies were $2.89 \pm 1.64$ semitones for $262 \mathrm{~Hz}$ (middle C; C4), $2.96 \pm 2.95$ semitones for $330 \mathrm{~Hz}(\mathrm{E} 4)$, and $3.08 \pm 2.55$ semitones for $391 \mathrm{~Hz}(\mathrm{G} 4)$. The mean pitch threshold for 42 adult CI subjects was $2.93 \pm 2.27$ semitones [Kang et al., 2009]. There was no significant difference between children and adults at any frequency.

\section{Melody Identification}

The children's performances on the isochronous melody identification test ranged from 2.78 to $25.0 \%$, and the average score was $10.61 \pm 7.74 \%$ (fig. $6 \mathrm{a}$ ). Statistical comparison between children and adults was not conducted because the children's performance was not significantly different from the chance level $(\mathrm{p}=0.35$; one-sample $\mathrm{t}$ test). 
Fig. 5. Complex pitch direction discrimination scores in UW-CAMP. Error bars indicate $1 \mathrm{SD}$. * Adult data $(\mathrm{n}=42)$ are adopted from Kang et al. [2009].
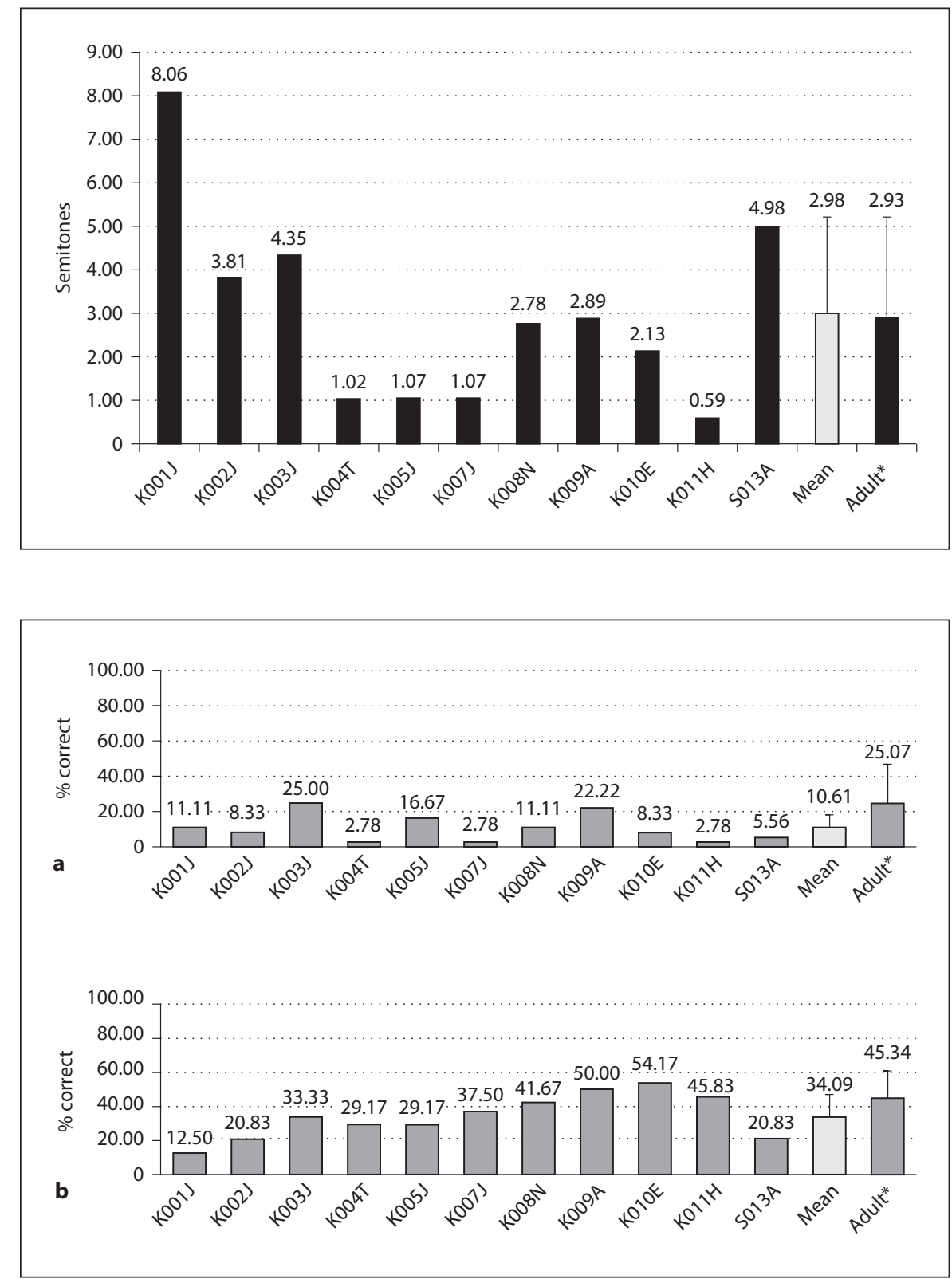

Fig. 6. a Melody identification scores. The children's melody scores were not significantly different from the chance level of $8.3 \%$ (one-sample t test, $\mathrm{p}=0.35$ ). b Timbre identification scores. The children's mean timbre scores were worse than those of the adults ( $\mathrm{t}$ test, $\mathrm{p}=0.039)$. Error bars indicate 1 SD. * Adult data $(n=42)$ adopted from Kang et al. [2009].

\section{Timbre Identification}

Timbre scores for implanted children ranged from 12.50 to $54.17 \%$ with a mean score of $34.09 \pm 13.15 \%$ (fig. 6b), which was significantly higher than the chance level ( $p=0.027)$. Kang et al. [2009] reported a mean timbre score of $45.34 \pm 16.21 \%$ correct for implanted adults. The children's timbre recognition scores were significantly worse than those of the adult subjects $(\mathrm{p}=0.039)$. The child subjects recognized the guitar most often (55\%) and the flute least often (15\%). Confusion matrices showed that the flute was most often confused with the violin.

Psychoacoustic and Clinical Outcomes in Prelingually Deafened CI Users

\section{Discussion}

In the present study, spectral-ripple discrimination, Schroeder-phase discrimination, speech perception in quiet and noise, and CAMP tests were successfully administered to prelingually deafened implanted children when they were appropriately instructed and encouraged. Their spectral-ripple discrimination, complexpitch direction discrimination, $\mathrm{CNC}$ word recognition, and speech perception in noise results were, within statistical bounds, similar to those reported for postlin- 
gually deafened adult CI users. However, they were generally poorer than adult CI users on Schroeder-phase discrimination as well as on melody and timbre identification.

Spectral abilities, as measured by spectral-ripple discrimination thresholds were statistically identical to those of adult CI users, but the child subjects were generally poor at the Schroeder-phase discrimination test and their scores were significantly worse than those of the adults. Previous studies demonstrated that temporal sensitivity takes longer to develop than frequency sensitivity, and normal-hearing children do not develop adult temporal processing ability until the age of 11 years [Wightman et al., 1989; Elfenbein et al., 1993; Buss et al., 1999]. In young normal-hearing listeners, peripheral temporal mechanisms may be adult-like, but the central auditory system may be less efficient in extracting temporal information [Plack and Moore, 1990]. It is possible then that the poorer Schroeder-phase discrimination scores seen in the children might be partly accounted for by the delayed maturation in their temporal processing ability.

Almost identical performance was observed in the two populations for CNC word recognition and spondee word recognition in steady-state noise. Prelingually implanted children and postlingually implanted adults were previously reported to have no difference in speech perception performance [Tyler et al., 2000; Oh et al., 2003]. In the present study, the same trend was also observed for complex-pitch direction discrimination. However, the children's melody and timbre scores were significantly worse than those of adults. This inconsistent pattern relative to adults' performance was also observed in the correlation analyses. For example, SRTs in steady noise were significantly correlated $(\mathrm{r}=-0.72, \mathrm{p}=0.018)$ with spectral-ripple discrimination thresholds in the child subjects (fig. 4), which is consistent with findings in adults [Won et al., 2007]. For music perception, spectral-ripple discrimination was not correlated with any of the three subtests, whereas adult CI users showed significant correlations between spectral-ripple discrimination and all three music subtests [Won et al., 2010]. It should be noted that correlation analyses with the melody scores are not appropriate in this study because the melody test results were at the chance level in the child subjects. It is not clear why correlation patterns are different in the two populations; however, the small sample size for the child subjects may certainly play a role. Familiarity with the musical instruments and melodies that make up the timbre and melody subtests should not be a significant factor as these children all were placed in educational settings that empha- sized musical exposure as part of their early education. They were nearly all mainstreamed by the time of testing.

However, it is reasonable to speculate that the different performance patterns observed in the children may be attributed to their immature temporal sensitivity and, as a result, they may use temporal and spectral sensitivities differently compared to adults. CI users receive limited information both for temporal and spectral aspects of sound. The limited temporal information is determined by the loss of temporal fine structure by implant sound processing and potentially limited envelope encoding at the electroneural interface. The limited number of spectral channels largely determines the limited spectral resolution in CI users. These device-related constraints are equally applied to both adults and children, but child CI users are further affected by their immature temporal sensitivity and potentially other limitations in the development of central temporal perception due to the degraded signal provided by the implant. Previous studies, either using acoustic vocoder simulation for normal-hearing listeners or manipulating the processing parameters for CI users, have demonstrated the relative importance of spectral and temporal sensitivity and their interaction on speech recognition [Cazals et al., 1994; Dooling et al., 2002; Smith et al., 2002; Stone et al., 2008]. For example, $\mathrm{Xu}$ et al. [2005] and Stone et al. [2008] showed that providing temporal envelope information is more beneficial for listeners when spectral resolution is limited. Using a different approach, Drennan et al. [2008] demonstrated the effect of interaction between spectral and temporal sensitivity of CI processing strategies. They tested two different processing strategies, HiResolution and Fidelity120, with the test batteries used in the present study. Fidelity120 showed better spectral-ripple discrimination, but worse Schroeder-phase discrimination compared to HiResolution. This spectrotemporal tradeoff resulted in the identical speech and music perception performance for the two strategies.

With this line of thought, it is plausible that the children's performance may have been driven more by their spectral sensitivity. The identical spectral-ripple thresholds obtained from the children and adults and the worse Schroeder-phase discrimination score in the children support this idea. Melody and timbre recognition may be determined by both spectral and temporal sensitivity, and the poorer temporal sensitivity in the children might have led them to perform worse than the adults. For speech perception, spectral sensitivity in the children may be sufficient to perform similarly to adults. This suggests that performance on musical tasks of prelingually 
deafened implanted children may improve after their temporal sensitivity has matured. This hypothesis can be tested in the future as this cohort grows older.

\section{Acknowledgements}

The authors are grateful for the dedicated efforts of all young subjects and their parents and guardians, and for the $\mathrm{NIH}$ grants R01-DC007525, P30-DC04661, L30-DC008490, and F31-DC009755, and an educational fellowship from Advanced Bionics Corporation.

\section{Disclosure Statement}

Dr. Rubinstein has been a paid consultant for Cochlear, Ltd. and Advanced Bionics and has received research funding from both companies. Neither company played any role in data acquisition, analysis, or the composition of the manuscript.

\section{References}

Bradham T, Jones J: Cochlear implant candidacy in the United States: prevalence in children 12 months to 6 years of age. Int J Pediatr Otorhinolaryngol 2008;7:1023-1028.

Buss E, Hall JW 3rd, Grose JH, Dev MB: Development of adult-like performance in backward, simultaneous, and forward masking. J Speech Lang Hear Res 1999;4:844-849.

-Cazals Y, Pelizzone M, Saudan O, Boex C: Lowpass filtering in amplitude modulation detection associated with vowel and consonant identification in subjects with cochlear implants. J Acoust Soc Am 1994;4:2048-2054.

-Chen JK, Chuang AY, McMahon C, Hsieh JC, Tung TH, Li LP: Music training improves pitch perception in prelingually deafened children with cochlear implants. Pediatrics 2010;4:e793-e800.

Dooling RJ, Leek MR, Gleich O, Dent ML: Auditory temporal resolution in birds: discrimination of harmonic complexes. J Acoust Soc Am 2002;2:748-759.

Drennan WR, Longnion JK, Ruffin C, Rubinstein JT: Discrimination of Schroeder-phase harmonic complexes by normal-hearing and cochlear-implant listeners. J Assoc Res Otolaryngol 2008;1:138-149.

Elfenbein JL, Small AM, Davis JM: Developmental patterns of duration discrimination. J Speech Hear Res 1993;4:842-849.

Fu QJ: Temporal processing and speech recognition in cochlear implant users. Neuroreport 2002;13:1635-1639.

Harris RW: Speech Audiometry Materials Compact Disk. Provo, Brigham Young University, 1991.

-Henry BA, Turner CW: The resolution of complex spectral patterns by cochlear implant and normal-hearing listeners. J Acoust Soc Am 2003;5:2861-2873.

- Henry BA, Turner CW, Behrens A: Spectral peak resolution and speech recognition in quiet: normal hearing, hearing impaired, and cochlear implant listeners. J Acoust Soc Am 2005;2:1111-1121.
Kang R, Nimmons GL, Drennan W, Longnion J, Ruffin C, Nie K, Won JH, Worman T, Yueh B, Rubinstein J: Development and validation of the University of Washington Clinical Assessment of Music Perception test. Ear Hear 2009;4:411-418.

Lauer AM, Molis M, Leek MR: Discrimination of time-reversed harmonic complexes by normal-hearing and hearing-impaired listeners. J Assoc Res Otolaryngol 2009;4:609619.

Levitt H: Transformed up-down methods in psychoacoustics. J Acoust Soc Am 1971;2:(suppl 2):467.

Nimmons GL, Kang RS, Drennan WR, Longnion J, Ruffin C, Worman T, Yueh B, Rubenstien JT: Clinical assessment of music perception in cochlear implant listeners. Otol Neurotol 2008;2:149-155.

Oh SH, Kim CS, Kang EJ, Lee DS, Lee HJ, Chang SO, Ahn SH, Hwang CH, Park HJ, Koo JW: Speech perception after cochlear implantation over a 4-year time period. Acta Otolaryngol 2003;2:148-153.

Oxenham AJ, Dau T: Towards a measure of auditory-filter phase response. J Acoust Soc Am 2001;6:3169-3178.

Peterson GE, Lehiste I: Revised CNC lists for auditory tests. J Speech Hear Disord 1962;27: 62-70.

Plack CJ, Moore BC: Temporal window shape as a function of frequency and level. J Acoust Soc Am 1990;5:2178-2187.

Saoji AA, Litvak L, Spahr AJ, Eddins DA: Spectral modulation detection and vowel and consonant identifications in cochlear implant listeners. J Acoust Soc Am 2009;3:955958.

Schroeder MR: Synthesis of low-peak-factor signals and binary sequences with low autocorrelation. IEEE Trans Inf Theory 1970; 16:85-89.

-Smith ZM, Delgutte B, Oxenham AJ: Chimaeric sounds reveal dichotomies in auditory perception. Nature 2002;6876:87-90.
Stone MA, Fullgrabe C, Moore BC: Benefit of high-rate envelope cues in vocoder processing: effect of number of channels and spectral region. J Acoust Soc Am 2008;4:22722282.

-Summers V: Effects of hearing impairment and presentation level on masking period patterns for Schroeder-phase harmonic complexes. J Acoust Soc Am 2000;5:2307-2317.

Summers V, Leek MR: Masking of tones and speech by Schroeder-phase harmonic complexes in normally hearing and hearing-impaired listeners. Hear Res 1998;1-2:139-150.

- Turner CW, Gantz BJ, Vidal C, Behrens A, Henry BA: Speech recognition in noise for cochlear implant listeners: benefits of residual acoustic hearing. J Acoust Soc Am 2004;4: 1729-1735.

- Tyler RS, Rubinstein JT, Teagle H, Kelsay D, Gantz BJ: Pre-lingually deaf children can perform as well as post-lingually deaf adults using cochlear implants. Cochlear Implants Int 2000;1:39-44.

Wightman F, Allen P, Dolan T, Kistler D, Jamieson D: Temporal resolution in children. Child Dev 1989;3:611-624.

-Won JH, Drennan WR, Kang RS, Rubinstein JT: Psychoacoustic abilities associated with music perception in cochlear implant users. Ear Hear 2010;6:796-805.

Won JH, Drennan WR, Rubinstein JT: Spectralripple resolution correlates with speech reception in noise in cochlear implant users. J Assoc Res Otolaryngol 2007;3:384-392.

-Won JH, Drennan WR, Nie K, Jameyson EM, Rubinstein JT: Acoustic temporal modulation detection and speech perception in cochlear implant listeners. J Acoust Soc Am 2011;130:376-388.

Xu L, Thompson CS, Pfingst BE: Relative contributions of spectral and temporal cues for phoneme recognition. J Acoust Soc Am 2005;5:3255-3267. 